\title{
Actitud hacia la masturbación en adolescentes: propiedades psicométricas de la versión española del Attitudes Toward Masturbation Inventory ${ }^{*}$
}

\author{
Attitudes Toward Masturbation in Adolescents: Psychometric Properties \\ of Spanish Version of Attitudes Toward Masturbation Inventory
}

Recibido: marzo 5 de 2009 | Revisado: junio 16 de 2009 | Aceptado: julio 12 de 2009

\author{
JuAN CARLOS SierRa \\ Universidad de Granada, Granada, España
}

FELIPE PERLA

Universidad de Oriente, El Salvador

RiCARDO GUTIÉRREZ-QUINTANILLA **

Universidad Tecnológica de El Salvador, El Salvador

Para citar este artículo. Sierra, J.C., Perla, F., \& Gutiérrez-Quintanilla, R. (2010). Actitud hacia la masturbación en adolescentes: propiedades psicométricas de la versión española del Attitudes Toward Masturbation Inventory. Universitas Psychologica, 9 (2), 531-542.

* Artículo de investigación en psicometría.

** Facultad de Psicología. Universidad de Granada. 18071 Granada, España. correo electrónico: jcsierra@ugr.es

RESUMEN

La masturbación es una conducta sexual frecuentemente asociada a la culpabilidad sexual. Ante la ausencia de instrumentos para evaluar las actitudes hacia esta conducta, este estudio pretende examinar algunas propiedades psicométricas de la versión española del Inventario de Actitudes Negativas hacia la Masturbación de Abramson y Mosher (IANM), el cual fue aplicado junto con la Encuesta de Opinión Sexual a una muestra de 610 adolescentes salvadoreños (308 varones y 302 mujeres). El análisis factorial exploratorio aisló tres factores: Afectos negativos en experiencias personales con la masturbación $(\alpha=0,85)$, Actitudes negativas hacia aspectos positivos de la masturbación $(\alpha=0,76)$ y Falsas creencias acerca de la naturaleza nociva de la masturbación $(\alpha=0,61)$. No se encontraron diferencias por sexo en la puntuación total de la escala ni en dos de las tres subescalas, pero sí en algunos de los ítems en los que las mujeres presentaban una actitud más negativa hacia la masturbación. La erotofilia se asoció inversamente a las actitudes negativas hacia la masturbación.

Palabras clave autores

Masturbación, actitudes, erotofilia, adolescentes, propiedades psicométricas. Palabras clave descriptores

Masturbación, psicología, mujeres adolescentes, actitudes, muchachos adolescentes, actitudes.

\section{A B S T R A C T}

Masturbation is a sexual behavior associated with sexuality guilt. In the absence of instruments to assess attitudes toward this behavior, this study aims to examine some psychometric properties of the Spanish version of Negative Attitudes Toward Masturbation Inventory (NAMI) from Abramson and Mosher, which was applied in conjuction whith the Sexual Opinion Survey to a sample of 610 Salvadorean adolescents (308 men and 302 women). The exploratory factorial analysis isolated three factors: Personally experienced negative affects associated with masturbation $(\alpha=0,85)$, Negative attitudes toward positive aspects of masturbation $(\alpha=0,76)$ and False beliefs about the harmful nature of masturbation $(\alpha=0,61)$. There were no sex differences in the total score of the scale or two of the three subscales, but in some of the items in which women had a more negative attitude toward masturbation. The 
erotophilia was inversely associated with negative attitudes toward masturbation.

Key words authors

Masturbation, Attitudes, Erotophilia, Adolescents, Psychometric Properties.

Key words plus

Masturbation, Psychology, Adolescent Girls, Attitudes, Adolescent Boys, Attitudes.

La masturbación ha sido, y sigue siendo, una conducta tabú para muchas personas. Desde la religión judeocristiana fue perseguida durante siglos por separarse del objetivo central de la sexualidad humana: la procreación. En el siglo XIX, desde posturas pseudocientíficas de la Medicina y Psiquiatría se la culpó de ser el origen de múltiples males del ser humano, tales como la depresión o psicosis delirantes. Posturas religiosas ortodoxas y distorsiones médicas tiñeron a esta conducta sexual de múltiples connotaciones negativas (Patton, 1986). Estos planteamientos, que calaron durante décadas entre la sociedad occidental, dieron lugar a que en múltiples ocasiones la masturbación se asociase a sentimientos de culpabilidad sexual (Abramson \& Mosher, 1975). Todavía, recientemente, Ortega, Ojeda, Sutil y Sierra (2005) ponen de manifiesto como las actitudes, que los adolescentes tienen hacia la masturbación, constituyen un factor fundamental en la explicación de la culpabilidad sexual.

El hecho de que la masturbación haya sido penalizada desde diversos sectores de influencia, no quiere decir que se trate de una conducta sexual rara e infrecuente. De hecho, Kinsey convulsionó a la tradicional sociedad estadounidense de mediados del siglo pasado, al afirmar que el 92\% de los hombres y el $62 \%$ de las mujeres se habían masturbado en alguna ocasión; la publicación de estos datos supuso, sin lugar a dudas, un importante cambio en la percepción y concepción de esta conducta. El último gran informe acerca de la conducta sexual de los estadounidenses (National Health and Social Life Survey) pone de manifiesto que el $61 \%$ de los varones y el $38 \%$ de las mujeres se habían masturbado en el último año (Das, 2007). Resultados similares fueron encontrados en otros países como Gran Bretaña (Gerressu, Mercer, Graham, Wellings \& Johnson, 2008) o España
(Lasheras Pérez, Cuñé Sala, Bautista Rodríguez \& Farré Martí, 2005; Malo de Molina, Valls Blanco \& Pérez Gómez, 1988; Van der Hofstadt, AntónRuiz, Tirado \& Navarro-Cremades, 2007).

En las últimas décadas, las posturas científicas hacia la masturbación cambiaron radicalmente y así, por ejemplo, desde los años setenta del pasado siglo se viene considerando su práctica como una herramienta útil en la terapia sexual de algunas disfunciones sexuales, como la inhibición del deseo sexual o la anorgasmia (Andersen, 1981; Kaplan, 1974; Riley \& Riley, 1978; Sierra \& Buela-Casal, 2001). Es más, en la actualidad se plantea el derecho al placer sexual, incluido el autoerotismo, como fuente de bienestar físico, psicológico, intelectual y espiritual (World Association for Sexual Health, 1999). No obstante, a pesar de los cambios experimentados en la concepción de la masturbación, todavía entre la población están presentes múltiples mitos, creencias erróneas y actitudes negativas hacia esta conducta sexual (Diéguez, Diz, Sueiro \& Chas, 2003; Gagnon, 1985; Miller \& Lief, 1976), constituyendo una causa de la culpabilidad sexual entre los adolescentes (Ortega et al., 2005). La asociación entre conocimientos sobre la masturbación y actitudes hacia la misma, está bien documentada (Miller \& Lief, 1976; Wang, Huang \& Lin, 2007), así como el efecto de éstas sobre la práctica de dicha conducta (Abramson \& Mosher, 1975; Lo Presto, Sherman \& Sherman, 1985; Wang et al., 2007). Por ello, tanto en terapia sexual como en la implementación de programas de educación sexual, es fundamental la evaluación de las actitudes hacia la masturbación. Cuesta entender, por ejemplo, que dentro del marco de intervención conductual en disfunciones sexuales se emplee la técnica de la masturbación sin evaluar previamente las actitudes hacia la misma. Pero incluso, aun no utilizando la masturbación como herramienta de mejora de la respuesta sexual, las actitudes hacia esta conducta son importantes, pues tal como demostraron Abramson, Perry, Rothblatt, Seeley y Seeley (1981), las actitudes positivas hacia la masturbación se asocian a un incremento en la vasocongestión pélvica durante la exposición a estímulos eróticos. La mayoría de programas de 
educación sexual para adolescentes incluyen entre sus contenidos la evaluación de conocimientos y actitudes hacia la masturbación, como es el caso del programa Agarimos (Carrera-Fernández, Lameiras-Fernández, Foltz, Núñez-Mangana \& Rodríguez-Castro, 2007; Lameiras Fernández, Rodríguez Castro, Ojea Rúa \& Dopereiro Rodríguez, 2004), aunque para tal objetivo no aporten ningún instrumento estandarizado.

A pesar de la importancia que tienen las actitudes hacia la masturbación en terapia y educación sexual, apenas existen autoinformes para su evaluación. Abramson y Mosher (1975) desarrollan el Negative Attitudes Toward Masturbation Inventory (Inventario de Actitudes Negativas hacia la Masturbación, IANM). Mediante análisis factorial con rotación varimax, aislaron tres factores que explicaron el $31,9 \%, 6,7 \%$ y $5,7 \%$ de la varianza total, respectivamente. El primer factor (Actitudes positivas hacia la masturbación) agrupaba a doce ítems (3, $4,5,11,13,14,17,18,20,22,27$ y 29), el segundo (Falsas creencias acerca de la naturaleza nociva de la masturbación) a diez $(1,2,6,7,9,15,16,21,24$ y 28) y el tercero (Afectos negativos en experiencias personales con la masturbación) a siete $(10,12,19$, $23,25,26$ y 30). A pesar de este planteamiento tridimensional, la fiabilidad de consistencia interna fue calculada para la escala total, informándose de un coeficiente de 0,75 mediante el método de las dos mitades (Abramson \& Mosher, 1975) y de un alfa de Cronbach de 0,94 (Mosher \& Vonderheide, 1985). Además de no aportarse en el estudio datos sobre la fiabilidad de consistencia interna de cada una de las dimensiones, tampoco se llevó a cabo un análisis estadístico de sus ítems. En cuanto a la validez de las medidas proporcionadas por el IANM, se informó que las actitudes negativas hacia la masturbación se asocian a una menor frecuencia masturbatoria y a un menor porcentaje de orgasmos durante la masturbación (Abramson \& Mosher, 1975), a una menor excitación subjetiva ante filmes de hombres y mujeres masturbándose (Mosher \& Abramson, 1977), a una menor vasocongestión pélvica durante la lectura de textos eróticos (Abramson et al., 1981) y a una mayor culpabilidad sexual (Abramson \& Mosher, 1975).
Por tanto, ante las evidencias de que la versión original del IANM constituye un instrumento fiable y valido, y ante la ausencia de autoinformes en lengua castellana que evalúen de manera específica las actitudes hacia la masturbación, este estudio instrumental (Montero \& León, 2007) se plantea llevar a cabo una adaptación del IANM. Para ello, y siguiendo las recomendaciones de Carretero-Dios y Perez (2007), se analizarán en una muestra de adolescentes las propiedades métricas de sus ítems, se examinará su estructura factorial y se aportarán algunas evidencias acerca de la validez sus medidas. Para este último objetivo, se analizarán, en primer lugar, las diferencias por sexo en la puntuación total de la escala, hipotetizando que no habrá diferencias significativas, tal como plantearon originalmente Abramson y Mosher (1975), aunque en algunos de sus ítems sí se encontrarán diferencias, como también se evidenció en el estudio original, presentando en este caso las mujeres una mayor puntuación, puesto que éstas se manifiestan más erotofóbicas que los hombres (Carpintero \& Fuertes, 1994; Geer \& Robertson, 2005; Lameiras \& Failde, 1998; Sierra, Ortega \& Gutiérrez-Quintanilla, 2008); en segundo lugar, se calculará la correlación de las puntuaciones del IANM con el constructo erotofilia, hipotetizando que las actitudes negativas hacia la masturbación correlacionarán en sentido negativo con la erotofilia.

\section{Método}

\section{Participantes}

La muestra seleccionada mediante un procedimiento no aleatorio estaba formada por 610 adolescentes salvadoreños (50,50\% varones y $49,50 \%$ mujeres), con edades comprendidas entre 14 y 18 años $(M=16,31 ; D T=0,87)$. Todos ellos eran estudiantes de dos instituciones de Educación Media de la ciudad de San Miguel (El Salvador) que cursaban diferentes opciones de Bachillerato: General (23\%), Comercio (40,7\%), Industrial $(23,1 \%)$ y Salud $(13,3 \%)$. Únicamente un $25,7 \%$ de los participantes informó haberse masturbado durante el último mes, siendo mucho mayor el 
porcentaje de varones $(46,9 \%)$ que el de mujeres (4\%) quienes lo habían hecho.

\section{Instrumentos}

- Inventario de Actitudes Negativas hacia la Masturbación (IANM; Abramson \& Mosher, 1975). Se empleó la versión de Mosher (1998) formada por 30 ítems contestados en una escala de respuesta con formato tipo Likert desde 1 (Totalmente falso) hasta 5 (Totalmente verdadero). Una vez invertidas las respuestas a 10 de sus ítems $(3,5,8,11,13,14,17,22,27$ y 29), su puntuación total oscila entre 30 y 150 , indicando puntuaciones elevadas mayor presencia de actitudes negativas hacia la masturbación. Sus propiedades psicométricas fueron descritas con anterioridad. La versión en español empleada en este estudio aparece en el Anexo.

- Encuesta de Opinión Sexual (Fisher, Byrne, White \& Kelley, 1988) que permite evaluar la dimensión erotofobia-erotofilia. Carpintero y Fuertes (1994) informan de una consistencia interna global de 0,86 para la adaptación española. Se empleó la adaptación salvadoreña de Sierra et al. (2008) formada por 16 ítems contestados en escala tipo Likert de siete puntos desde 1 (Totalmente en desacuerdo) a 7 (Totalmente de acuerdo), indicando las puntuaciones elevadas un mayor grado de erotofilia. El análisis factorial exploratorio llevado a cabo una vez eliminados 5 de los 21 ítems originales que no alcanzaban valores adecuados en su correlación con la escala total, dio lugar a un único factor (erotofilia) que presenta un coeficiente de fiabilidad de 0,84; por lo que respecta a la validez de la escala, la erotofilia se asoció con ser varón, tener pareja, orientación bisexual, residir en poblaciones grandes, escasa práctica religiosa e ideología de izquierda (Sierra et al., 2008).

\section{Procedimiento}

En primer lugar, se hizo una traducción al español de los ítems del Attitudes towards Masturbation
Inventory, la cual fue contrastada con la versión original por dos traductores que mostraron un acuerdo completo con la traducción de cada uno de los ítems. La versión traducida fue revisada por cinco psicólogos expertos en sexualidad humana, con el objetivo de que indicasen si la redacción de cada uno de sus ítems era correcta o no; a continuación, se calculó para cada ítem el porcentaje de acuerdo, corrigiendo aquellos que no alcanzaron el $85 \%$ de acuerdo, a partir de los sugerencias de los cinco expertos. Esta nueva versión del instrumento fue administrada a un grupo de veinticinco adolescentes, los cuales indicaron, después de cada ítem, si éste era comprensible o no, calculándose a continuación el porcentaje de acuerdos y corrigiendo aquellos ítems que no alcanzaron un 85\% de acuerdo, dando lugar a la versión definitiva del instrumento.

En segundo lugar, se realizó un contacto telefónico y por escrito con los directores de las dos instituciones educativas seleccionadas para el estudio para concertar una entrevista, durante la cual se les explicaron los objetivos de la investigación. Una vez obtenidos los permisos correspondientes de padres y maestros, se procedió a la administración de la prueba, la cual fue llevada a cabo de forma colectiva en las aulas, por un solo evaluador, a lo largo de nueve días. Todos los participantes recibieron las mismas instrucciones, asegurándoseles que sus respuestas serían totalmente anónimas. Ningún adolescente rehusó colaborar en el estudio.

\section{Análisis estadísticos}

Primero se llevó a cabo un análisis estadístico de los ítems del IANM calculando su media, desviación típica, correlación ítem-total y alfa de Cronbach si el ítem es eliminado. A continuación, con la finalidad de analizar la estructura del instrumento, se realizó un análisis factorial exploratorio, de componentes principales, con rotación varimax, prefijando tres factores, tal como se propone en la solución factorial original de Abramson y Mosher (1975). Para la estimación de la fiabilidad de consistencia interna se calculó el alfa de Cronbach. 
Con el objetivo de obtener evidencias acerca de la validez de las medidas del IANM, en primer lugar, se calcularon las diferencias por sexo, mediante la prueba de Mann-Whitney, tanto para las puntuaciones de la escala total y de las tres subescalas, como para la de cada ítem, tal como se había realizado en el estudio original y, en segundo lugar, se correlacionaron las puntuaciones del IANM con el costructo erotofilia.

\section{Resultados}

En primer lugar, se llevó a cabo un análisis de las propiedades métricas de los ítems y de la consistencia interna de la escala total. Tal como se aprecia en la Tabla 1, la media de respuesta a los reactivos osciló entre 2,50 (ítem 3) y 3,68 (ítem 5), localizándose la respuesta media por debajo del punto medio de la escala de respuesta únicamente en nueve de los treinta ítems $(3,7,8,12,14,17,23$, 27 y 29). Los valores de las desviaciones típicas se situaron ligeramente por encima de uno (entre 1,35 del ítem 16 y 1,58 del ítem 4), tal como es recomendable (Carretero-Dios \& Pérez, 2005). En cuanto a las correlaciones entre la puntuación del ítem y la puntuación total, y considerando como valores adecuados aquellos que son superiores a 0,25 (Nunnally \& Berstein, 1995), encontramos un número considerable de ítems $(1,3,5,8,11$, $13,14,17,22,27$ y 29) que se sitúan por debajo de ese límite, presentando el valor más alto el ítem $18(0,45)$. No obstante, teniendo en cuenta que la eliminación de algunos de estos ítems $(3,8,11$, $13,14,17,22,27$ y 29) supondría un incremento muy poco relevante en el coeficiente de consistencia interna de la escala total -el cual es bastante moderado $(\alpha=0,61)$-, en este primer momento se descartó la opción de eliminar ítems, a la espera de examinar sus propiedades psicométricas dentro de su correspondiente subescala, una vez realizado el análisis factorial.

En segundo lugar, y después del análisis estadístico de los ítems, se llevó a cabo un análisis de componentes principales, rotación varimax, prefijando tres factores. Previamente, tanto el test de Kaiser-Meyer-Olkin $(\mathrm{KMO}=0,90)$ como la

\section{TABLA 1}

Medias (M), desviaciones típicas (DT), correlación ítem-total $\left(\mathrm{r}_{\mathrm{i}-\mathrm{t}}\right)$ y alfa de Cronbach si el ítem es eliminado $(\alpha)$

\begin{tabular}{ccccc}
\hline Ítem & $\mathbf{M}$ & $\mathrm{DT}$ & $\mathbf{r}_{\mathrm{i}-\mathrm{t}}$ & $\alpha$ \\
\hline 1 & 3,07 & 1,38 & 0,14 & 0,60 \\
2 & 3,15 & 1,36 & 0,25 & 0,59 \\
3 & 2,50 & 1,44 & $-0,09$ & 0,62 \\
4 & 3,42 & 1,58 & 0,34 & 0,58 \\
5 & 3,68 & 1,42 & 0,03 & 0,61 \\
6 & 3,10 & 1,43 & 0,27 & 0,59 \\
7 & 2,81 & 1,45 & 0,26 & 0,59 \\
8 & 2,87 & 1,46 & $-0,27$ & 0,64 \\
9 & 3,03 & 1,50 & 0,33 & 0,58 \\
10 & 3,07 & 1,44 & 0,28 & 0,59 \\
11 & 3,15 & 1,37 & $-0,22$ & 0,64 \\
12 & 2,95 & 1,49 & 0,33 & 0,58 \\
13 & 3,04 & 1,45 & $-0,14$ & 0,63 \\
14 & 2,79 & 1,43 & $-0,17$ & 0,63 \\
15 & 3,36 & 1,37 & 0,35 & 0,58 \\
16 & 3,19 & 1,35 & 0,33 & 0,58 \\
17 & 2,95 & 1,38 & $-0,11$ & 0,63 \\
18 & 3,25 & 1,51 & 0,45 & 0,57 \\
19 & 3,28 & 1,40 & 0,41 & 0,57 \\
20 & 3,09 & 1,46 & 0,36 & 0,58 \\
21 & 3,21 & 1,42 & 0,30 & 0,58 \\
22 & 3,30 & 1,43 & $-0,08$ & 0,62 \\
23 & 2,81 & 1,44 & 0,36 & 0,58 \\
24 & 3,01 & 1,40 & 0,38 & 0,58 \\
25 & 3,38 & 1,50 & 0,37 & 0,58 \\
26 & 3,38 & 1,46 & 0,39 & 0,57 \\
27 & 2,77 & 1,41 & $-0,14$ & 0,63 \\
28 & 3,27 & 1,40 & 0,31 & 0,58 \\
29 & 2,97 & 1,42 & $-0,13$ & 0,63 \\
30 & 3,14 & 1,46 & 0,40 & 0,57 \\
\hline & & & &
\end{tabular}

Fuente: elaboración propia.

prueba de esfericidad de Bartlett $\left(\chi_{435}^{2}=4270,94\right.$; $p<0,001)$ aseguraron la idoneidad de los datos para la realización de dicho análisis. La solución trifactorial forzada explicó un 35,38\% de la varianza (véase la Tabla 2). El primer factor, denominado Afectos negativos en experiencias personales con la masturbación, agrupó un total de 14 ítems $(10,12,15,16,18,19,20,21,23,24,25,26,28$ y $30)$, siete de los cuales coinciden con la subescala original de Mosher y Anderson (1975), llegando a explicar el $15,26 \%$ de la varianza total, con un valor propio de 4,58. Las saturaciones en este fac- 
tor oscilaron entre 0,30 del ítem 10 (Después de la masturbación, la persona se siente degradada) y 0,63 del ítem 30 (Después de masturbarme me disgusto por la falta de control sobre mi cuerpo). La fiabilidad de consistencia interna de este primer factor fue de 0,85 , presentando todos sus ítems unas propiedades métricas adecuadas: medias en torno al punto intermedio de la escala de respuesta, desviaciones típicas ligeramente superiores a uno y adecuados índices de discriminación (véase la Tabla 3).

El segundo factor, denominado Actitudes negativas hacia aspectos positivos de la masturbación, incluye 10 ítems $(3,5,8,11,13,14,17,22,27 \mathrm{y}$ 29), nueve de los cuales coinciden con la subescala original Actitudes positivas hacia la masturbación y explica el 11,14\% de la varianza total, con un valor propio de 3,34. Las cargas factoriales de sus ítems oscilaron entre 0,21 del ítem 8 (La masturbación excesiva es físicamente imposible, una idea absurda) y 0,63 de ítem 29 (La masturbación puede enseñar a disfrutar la sensibilidad del propio cuerpo) (véase la Tabla 2). Esta subescala alcanzó un coeficiente de consistencia interna (alfa de Cronbach) de 0,76, presentando todos sus ítems valores medios de respuesta en torno al punto medio de la escala de respuesta, desviaciones típicas por encima de uno y valores de correlación ítem-resto superiores a 0,25 , tal como recomiendan Nunnally y Bernstein (1995) (véase la Tabla 3).

El tercer factor integró a 6 ítems $(1,2,4,6,7$ y 9) que, a excepción del 4, coinciden con la subescala original denominada Falsas creencias acerca de la naturaleza nociva de la masturbación. Este factor explicó un 8,98\% de la varianza, con un valor propio de 2,69. Sus ítems presentaron saturaciones superiores a 0,40, oscilando entre 0,41 del ítem 1 (La gente se masturba para escapar de sentimientos de tensión e inquietud) y 0,55 del ítem 2 (La gente que se masturba no disfruta tanto del coito) (véase la Tabla 2). El coeficiente alfa de Cronbach de esta subescala fue inferior al de las otras dos, obteniendo un valor de 0,61. A su vez, todos los ítems que la componen presentan propiedades métricas adecuadas (véase la Tabla 3).

En tercer lugar, y con el objetivo de aportar evidencias acerca de la validez teórica de las me-
TABLA 2

Matriz de estructura del análisis de componentes principales con rotación varimax

\begin{tabular}{|c|c|c|c|}
\hline Ítems & F1 & $F 2$ & F3 \\
\hline 30 & $0,63 *$ & & \\
\hline 26 & $0,62 *$ & & \\
\hline 25 & $0,61 *$ & & \\
\hline 24 & 0,60 & & \\
\hline 23 & $0,57^{*}$ & & \\
\hline 28 & 0,57 & & \\
\hline 19 & $0,54 *$ & & 0,27 \\
\hline 18 & 0,54 & & 0,26 \\
\hline 21 & 0,47 & & \\
\hline 20 & 0,46 & & 0,28 \\
\hline 15 & 0,46 & & 0,31 \\
\hline 16 & 0,45 & & 0,25 \\
\hline 12 & $0,44^{*}$ & & \\
\hline 29 & & $0,63 *$ & \\
\hline 27 & & $0,60 *$ & \\
\hline 17 & & $0,60 *$ & \\
\hline 22 & & $0,57 *$ & \\
\hline 3 & & $0,57 *$ & \\
\hline 13 & & $0,56^{*}$ & \\
\hline 5 & & $0,49 *$ & \\
\hline 14 & & $0,45^{*}$ & \\
\hline 11 & & $0,35 *$ & \\
\hline 2 & & & $0,55^{*}$ \\
\hline 8 & & 0,21 & \\
\hline 4 & 0,21 & & 0,53 \\
\hline 7 & & & $0,52 *$ \\
\hline 6 & & & $0,52 *$ \\
\hline 9 & 0,29 & & $0,49 *$ \\
\hline 1 & & & $0,41 *$ \\
\hline 10 & $0,30 *$ & & 0,34 \\
\hline Autovalor & 4,58 & 3,34 & 2,69 \\
\hline $\begin{array}{c}\% \text { de } \\
\text { varianza }\end{array}$ & 15,26 & 11,14 & 8,98 \\
\hline
\end{tabular}

Notas: Para mayor claridad de la tabla se han eliminado las saturaciones inferiores a 0,20. F1: Afectos negativos en experiencias personales con la masturbación; F2: Actitudes negativas hacia aspectos positivos de la masturbación; F3: Falsas creencias acerca de la naturaleza nociva de la masturbación. En negrita aparece la saturación en el factor al que ha sido asignado cada uno de los ítems. * Ítems que coinciden con la distribución factorial original de Abramson y Mosher (1975).

Fuente: elaboración propia. 
TABLA 3

Medias (M), desviaciones típicas (DT), correlación ítem-total $\left(\mathrm{r}_{\mathrm{i}-\mathrm{t}}\right)$ y alfa de Cronbach si el ítem es eliminado $(\alpha)$ en cada factor por separado

\begin{tabular}{|c|c|c|c|c|}
\hline \multicolumn{5}{|c|}{ F1 $(\alpha=0,85)$} \\
\hline Ítem & M & DT & $r_{i-t}$ & $\alpha$ \\
\hline 30 & 3,14 & 1,46 & 0,53 & 0,83 \\
\hline 26 & 3,39 & 1,46 & 0,52 & 0,83 \\
\hline 25 & 3,39 & 1,50 & 0,49 & 0,84 \\
\hline 24 & 3,01 & 1,41 & 0,52 & 0,83 \\
\hline 23 & 2,81 & 1,44 & 0,49 & 0,84 \\
\hline 28 & 3,28 & 1,40 & 0,53 & 0,83 \\
\hline 19 & 3,28 & 1,40 & 0,51 & 0,83 \\
\hline 18 & 3,25 & 1,51 & 0,50 & 0,84 \\
\hline 21 & 3,21 & 1,42 & 0,47 & 0,84 \\
\hline 20 & 3,09 & 1,46 & 0,48 & 0,84 \\
\hline 15 & 3,37 & 1,37 & 0,48 & 0,84 \\
\hline 16 & 3,19 & 1,35 & 0,49 & 0,84 \\
\hline 12 & 2,95 & 1,49 & 0,40 & 0,84 \\
\hline 10 & 3,07 & 1,44 & 0,39 & 0,84 \\
\hline \multicolumn{5}{|c|}{$\mathrm{F} 2(\alpha=0,76)$} \\
\hline Ítem & M & DT & $r_{i-t}$ & $\alpha$ \\
\hline 29 & 2,97 & 1,42 & 0,53 & 0,73 \\
\hline 27 & 2,77 & 1,41 & 0,48 & 0,73 \\
\hline 17 & 2,95 & 1,38 & 0,48 & 0,73 \\
\hline 22 & 3,30 & 1,43 & 0,43 & 0,74 \\
\hline 3 & 2,50 & 1,45 & 0,41 & 0,74 \\
\hline 13 & 3,04 & 1,45 & 0,48 & 0,73 \\
\hline 5 & 3,68 & 1,42 & 0,29 & 0,76 \\
\hline 14 & 2,79 & 1,43 & 0,47 & 0,73 \\
\hline 11 & 3,15 & 1,37 & 0,38 & 0,75 \\
\hline 8 & 2,87 & 1,42 & 0,28 & 0,76 \\
\hline \multicolumn{5}{|c|}{ F3 $(\alpha=0,61)$} \\
\hline Ítem & $M$ & DT & $r_{i-t}$ & $\alpha$ \\
\hline 2 & 3,15 & 1,36 & 0,41 & 0,54 \\
\hline 4 & 3,42 & 1,58 & 0,37 & 0,56 \\
\hline 7 & 2,81 & 1,45 & 0,32 & 0,58 \\
\hline 6 & 3,10 & 1,43 & 0,34 & 0,57 \\
\hline 9 & 3,04 & 1,50 & 0,35 & 0,57 \\
\hline 1 & 3,07 & 1,38 & 0,28 & 0,59 \\
\hline
\end{tabular}

Notas: F1: Afectos negativos en experiencias personales con la masturbación; F2: Actitudes negativas hacia aspectos positivos de la masturbación; F3: Falsas creencias acerca de la naturaleza nociva de la masturbación. Los ítems de cada subescala aparecen ordenados en función de sus saturaciones en la subescala.

Fuente: elaboración propia. didas del IANM, se compararon entre varones y mujeres las puntuaciones en la escala total, en las tres subescalas y en cada uno de los ítems. En la puntuación total no se encontraron diferencias estadísticamente significativas entre varones $(M$ $=92,66 ; D T=12,82)$ y mujeres $(M=93,37$; $D T=11,60)(Z=-0,95 ; p=0,343)$. En cuanto a las subescalas, no se encontraron diferencias en afectos negativos en experiencias personales con la masturbación $(Z=-1,62 ; p=0,105)$ ni en falsas creencias acerca de la naturaleza nociva de la masturbación $(Z=-1,74 ; p=0,082)$; en cambio, sí se obtuvieron diferencias en actitudes negativas hacia aspectos positivas de la masturbación $(Z=$ 3,20; $p<0,01$ ), presentando mayor puntuación las mujeres $(M=31,09 ; D T=8,44)$ que los varones $(M=28,99$; $D T=7,43)$. Si se analizan estas diferencias por sexo ítem a ítem, se aprecia que en 13 de los 30 ítems se encuentran diferencias estadísticamente significativas (véase la Tabla 4). Así, las mujeres presentan mayores puntuaciones en los ítems 5 (La masturbación en la niñez puede ayudar a desarrollar una actitud saludable y natural hacia el sexo), 7 (La masturbación puede conducir a la homosexualidad), 9 (Si disfruta mucho masturbándose, no aprenderá nunca a relacionarse con el sexo opuesto), 13 (La masturbación puede ser el "amigo en la necesidad" cuando no hay "amigo en el acto"), 17 ( $\mathrm{La}$ masturbación puede proporcionar un alivio inofensivo a la tensión sexual), 18 (Jugar con los propios genitales es repugnante), 22 (La masturbación es divertida), 27 (La masturbación es un desahogo sexual normal) y 29 (La masturbación puede enseñar a disfrutar la sensibilidad del propio cuerpo), mientras que éstos las superan únicamente en los ítems 10 (Después de la masturbación la persona se siente degradada), 23 (Cuando me masturbo, me disgusto conmigo mismo), 28 (La masturbación es ocasionada por una excesiva preocupación por el sexo) y 30 (Después de masturbarme me disgusto por la falta de control sobre mi cuerpo).

Por último, el constructo erotofilia correlacionó de forma significativa en sentido negativo con actitudes negativas hacia aspectos positivas de la masturbación $(r=-0,28 ; p<0,001)$ y falsas creencias acerca de la naturaleza nociva de la masturbación $(r=-0,16 ; p<0,001)$. 
TABLA 4

Análisis de las diferencias por sexo para cada uno de los ítems del IANM

\begin{tabular}{|c|c|c|c|c|}
\hline & $\begin{array}{l}\text { Varones } \\
(\mathrm{n}=308)\end{array}$ & $\begin{array}{c}\text { Mujeres } \\
(\mathrm{n}=302)\end{array}$ & & \\
\hline Ítem & $\mathrm{M}(\mathrm{DT})$ & $\mathrm{M}(\mathrm{DT})$ & Z & $\mathrm{P}$ \\
\hline 1 & $3,06(1,40)$ & $3,07(1,36)$ & $-0,08$ & 0,938 \\
\hline 2 & $3,22(1,32)$ & $3,08(1,40)$ & $-1,26$ & 0,206 \\
\hline 3 & $2,47(1,40)$ & $2,54(1,49)$ & $-0,41$ & 0,679 \\
\hline 4 & $3,43(1,56)$ & $3,40(1,60)$ & $-0,11$ & 0,912 \\
\hline 5 & $3,57(1,41)$ & $3,79(1,43)$ & $-2,26$ & $0,024^{*}$ \\
\hline 6 & $3,05(1,45)$ & $3,16(1,42)$ & $-0,96$ & 0,339 \\
\hline 7 & $2,66(1,48)$ & $2,97(1,40)$ & $-2,75$ & $0,006 * *$ \\
\hline 8 & 2,94 $(1,46)$ & $2,80(1,46)$ & $-1,23$ & 0,218 \\
\hline 9 & $2,88(1,51)$ & $3,20(1,48)$ & $-2,62$ & $0,009 * *$ \\
\hline 10 & $3,24(1,42)$ & $2,89(1,43)$ & $-3,06$ & $0,002 * *$ \\
\hline 11 & $3,07(1,34)$ & $3,23(1,40)$ & $-1,52$ & 0,129 \\
\hline 12 & $3,07(1,45)$ & $2,83(1,52)$ & $-1,93$ & 0,054 \\
\hline 13 & $2,91(1,43)$ & $3,17(1,46)$ & $-2,19$ & $0,029 *$ \\
\hline 14 & $2,79(1,40)$ & $2,79(1,47)$ & $-0,07$ & 0,941 \\
\hline 15 & $3,35(1,34)$ & $3,38(1,41)$ & $-0,51$ & 0,613 \\
\hline 16 & $3,22(1,30)$ & $3,17(1,41)$ & $-0,35$ & 0,722 \\
\hline 17 & 2,81 $(1,29)$ & $3,09(1,45)$ & $-2,45$ & $0,014 *$ \\
\hline 18 & $3,14(1,46)$ & $3,36(1,55)$ & $-2,07$ & $0,039 *$ \\
\hline 19 & $3,29(1,36)$ & $3,27(1,45)$ & $-0,004$ & 0,997 \\
\hline 20 & $3,14(1,43)$ & $3,03(1,49)$ & $-0,87$ & 0,387 \\
\hline 21 & $3,30(1,35)$ & $3,12(1,48)$ & $-1,32$ & 0,185 \\
\hline 22 & $3,07(1,41)$ & $3,53(1,42)$ & $-4,13$ & $0,000 * * *$ \\
\hline 23 & $2,97(1,42)$ & $2,65(1,44)$ & $-2,82$ & $0,005 * *$ \\
\hline 24 & $3,06(1,36)$ & $2,97(1,45)$ & $-0,80$ & 0,421 \\
\hline 25 & $3,36(1,43)$ & $3,41(1,57)$ & $-0,81$ & 0,419 \\
\hline 26 & $3,45(1,40)$ & $3,32(1,51)$ & $-0,89$ & 0,375 \\
\hline 27 & $2,59(1,35)$ & 2,95 $(1,44)$ & $-3,13$ & $0,002 * *$ \\
\hline 28 & $3,43(1,33)$ & $3,12(1,46)$ & $-2,57$ & $0,010 *$ \\
\hline 29 & $2,76(1,37)$ & $3,19(1,44)$ & $-3,74$ & $0,000 * * *$ \\
\hline 30 & $3,41(1,41)$ & $2,86(1,45)$ & $-4,72$ & $0,000 * * *$ \\
\hline
\end{tabular}

*** $p<0,001 ; * * p<0,01 ; * * p<0,05$

Fuente: elaboración propia.

\section{Discusión}

Apenas existen instrumentos para evaluar las actitudes hacia la masturbación, a pesar de tratarse de una conducta sexual de suma importancia en el estudio de la sexualidad humana. El análisis de las actitudes hacia esta conducta se hace necesario cuando para algunas personas su práctica puede ser una fuente importante de culpabilidad sexual (Ortega et al., 2005) o cuando se plantea su uso en terapia sexual (Sierra \& Buela-Casal, 2001). En este estudio, se analizaron algunas propiedades psicométricas del Inventario de Actitudes Negativas hacia la Masturbación de Abramson y Mosher (1975) en una muestra de adolescentes. El análisis factorial exploratorio llevado a cabo mostró una estructura de tres factores muy similar a la propuesta de los autores de la escala. El primero, Afectos negativos en experiencias personales con la masturbación, agrupó a 14 ítems que describen experiencias personales negativas con la masturbación, como degradación (ítem 10), culpa (ítem 12), repugnancia (ítem 18), compulsión (ítem 21), disgusto (ítems 23 y 30) o vergüenza (ítem 25), asî como consecuencias asociadas a experiencias con la misma, tales como inmadurez sexual (ítem 16), trastornos de la conducta (ítem 19), aislamiento social (ítem 24) o agotamiento mental (ítem 26). De estos catorce ítems, siete coinciden con el factor original de Abramson y Mosher, en concreto, el 10, 12, 19, 23, 25, 26 y 30. El segundo factor, denominado Actitudes negativas hacia aspectos positivos de la masturbación, coincide prácticamente con el de actitudes positivas hacia la masturbación planteado por los autores de la escala. Este factor agrupa a 10 ítems que describen aspectos positivos de la masturbación y cuya puntuación estaría reflejando una valoración negativa de los mismos; entre estos aspectos positivos estaría el desarrollo de actitudes sexuales saludables (ítem 5), la facilitación del orgasmo femenino (ítem 11), la diversión (ítem 22), el alivio de la tensión sexual (ítems 14, 17 y 27) o el aprendizaje de la sensibilidad del propio cuerpo (ítem 29). Por último, el tercer factor, Falsas creencias acerca de la naturaleza nociva de la masturbación, agrupó a seis ítems, cinco de los cuales formaban parte del factor original de Abramson y Mosher (1975). Estos cinco ítems se refieren a valoraciones negativas de la masturbación como, por ejemplo, que se trata de un pecado (ítem 4) o de una conducta inmadura (ítem 6), o consecuencias de la misma, pero más generales y no asociadas en principio a experiencias personales como ocurría con los ítems del factor 1 , como menor disfrute del coito 
(ítem 2), homosexualidad (ítem 7) o imposibilidad de mantener relaciones heterosexuales (ítem 9).

A diferencia del estudio original de Abramson y Mosher (1975), se llevó a cabo un análisis estadístico de los ítems que componen cada uno de los factores. Los resultados encontrados indicaron que todos ellos presentan adecuadas propiedades métricas dentro de cada factor. Así, tal como sugieren Carretero-Dios y Pérez (2005), la media de respuesta a cada ítem se sitúa en torno al punto medio teórico de la escala de respuesta, las desviaciones típicas están por encima de uno, las correlaciones ítem-resto presentan valores superiores a 0,25 y la eliminación de ítems no supone ninguna mejora de la fiabilidad de consistencia interna en cada una de las tres subescalas, las cuales presentan valores satisfactorios en los tres casos. Si tenemos en cuenta la media de respuesta a cada ítem, se puede apreciar como en la mayoría de ellos (a excepción de nueve), ésta se sitúa por encima del punto medio teórico de la escala de respuesta (tres), lo cual está indicando que, en promedio, en la mayoría de ítems, los adolescentes de la muestra evaluada manifiestan actitudes negativas hacia la masturbación. En el estudio de Abramson y Mosher, los ítems con una media de respuesta superior a tres eran 17, lo que está indicando que las diferencias de respuestas entre la muestra de estudiantes universitarios empleada en el estudio original y la de adolescentes del presente estudio son escasas.

En cuanto a los indicadores de validez examinados, tal como se había hipotetizado, y siguiendo los resultados de Abramson y Mosher (1975), no se encontraron diferencias estadísticamente significativas entre varones y mujeres en la puntuación total del IANM ni en dos de sus subescalas; sin embargo, sí se apreciaron diferencias en el factor Actitudes negativas hacia aspectos positivos de la masturbación, en donde las mujeres presentaron una mayor puntuación. Esta misma tendencia se aprecia al analizar los ítems de forma individual. Así, en trece de los treinta ítems existen diferencias significativas por sexo, mostrando las mujeres en nueve de ellos una mayor puntuación, es decir, más actitudes negativas hacia la masturbación. Únicamente, los hombres superan a las mujeres en cua- tro ítems referidos a experiencias personales con la masturbación: degradación, disgusto, excesiva preocupación sexual y falta de autocontrol. Teniendo en cuenta que es mucho mayor el porcentaje de varones que el de mujeres que informa haberse masturbado en el último mes (46,9\% frente al $4 \%$ ), parece lógico pensar que los primeros puedan superar a las segundas en ítems que tienen que ver con experiencias personales con la masturbación. Estos resultados ratifican que las mujeres manifiestan posiciones más erotofóbicas que los varones, tal como ya habían señalado otros estudios (Carpintero \& Fuertes, 1994; Geer \& Robertson, 2005; Lameiras \& Failde, 1998; Sierra et al., 2008). Esta asociación entre actitudes hacia la masturbación y actitudes sexuales en general se aprecia a su vez en la correlación negativa obtenida (baja, pero significativa) entre erotofilia y dos de las subescalas del IANM.

En definitiva, la versión española del IANM presenta indicios adecuados de fiabilidad y validez que le convierten en un instrumento útil para la evaluación de las actitudes hacia la masturbación en adolescentes y cuyo empleo será interesante en la implementación de programas de educación sexual. Futuros estudios deben ratificar estos primeros resultados, ampliando su utilidad a otros contextos y poblaciones, como por ejemplo, adultos con disfunciones sexuales, de cara a su empleo en terapia sexual.

\section{Referencias}

Abramson, P. R. \& Mosher, D. L. (1975). Development of a measure of negative attitudes toward masturbation. Journal of Consulting and Clinical Psychology, 43, 485-490.

Abramson, P. R., Perry, L. B., Rothblatt, A., Seeley, T. T \& Seeley, D. M. (1981). Negative attitudes toward masturbation and pelvic vasocongestion: A thermographic analysis. Journal of Research in Personality, 15, 497-509.

Andersen, B. L. (1981). A comparison of systematic desensitization and directed masturbation in the treatment of primary orgasmic dysfunction in females. Journal of Consulting and Clinical Psychology, 49, 568-570. 
Carpintero, E. \& Fuertes, A. (1994). Validación de la versión castellana del "Sexual Opinion Survey" (SOS). Cuadernos de Medicina Psicosomática, 31, 52-61.

Carrera-Fernández, M. V., Lameiras-Fernández, M., Foltz, M. L., Núñez-Mangana, A. M. \& RodríguezCastro, Y. (2007). Evaluación de un programa de educación sexual con estudiantes de Educación Secundaria Obligatoria. International Journal of Clinical and Health Psychology, 7, 739-751.

Carretero-Dios, H. \& Pérez, C. (2005). Normas para el desarrollo y revisión de estudios instrumentales. International Journal of Clinical and Health Psychology, 5, 521-551.

Carretero-Dios, H. \& Pérez, C. (2007). Standards for the development and review of instrumental studies: Considerations about test selection in psychological research. International Journal of Clinical and Health Psychology, 7, 863-882.

Fisher, W. A., Byrne, D., White, L. A. \& Kelley, K. (1988). Erotophobia-erotophilia as a dimension of personality. Journal of Sex Research, 25, 123-151.

Das, A. (2007). Masturbation in the United Status. Journal of Sex and Marital Therapy, 33, 301-317.

Diégez, J. L., Diz, M. C., Sueiro, E. \& Chas M. D. (2003). Actitudes hacia la sexualidad de adolescentes

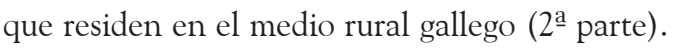
Cuadernos de Medicina Psicosomática y Psiquiatría de Enlace, 66, 56-67.

Gagnon, J. K. H. (1985). Attitudes and responses of parents to pre-adolescent masturbation. Archives of Sexual Behavior, 14, 451-509.

Geer, J. \& Robertson, G. G. (2005). Implicit attitudes in sexuality: Gender differences. Archives of Sexual Behavior, 34, 671-677.

Gerressu, M., Mercer, C. H., Graham, C. A., Wellings, K. \& Johnson, A. M. (2008). Prevalence of masturbation and associated factors in a British national probability survey. Archives of Sexual Behavior, 37, 266-278.

Kaplan, H. (1974). The new sex therapy. Nueva York: Bruner and Mazel.

Lasheras Pérez, M. G., Cuñé Sala, J., Bautista Rodríguez, C. \& Farré Martí, J. M. (2005). Hábitos sexuales en jóvenes universitarios. Cuadernos de Medicina Psicosomática y Psiquiatría de Enlace, 74, 57-63.
Lameiras, M. \& Failde, J. (1998). Sexualidad y salud en jóvenes universitarios/as: actitudes, actividad sexual y percepción de riesgo de la transmisión heterosexual del VIH. Análisis y Modificación de Conducta, 23, 27-63.

Lameiras Fernández, M., Rodríguez Castro, Y., Ojea Rúa, M. \& Dopereiro Rodríguez, M. (2004). Programa Agarimos. Programa coeducativo de desarrollo psicoafectivo y sexual. Madrid: Pirámide.

Lo Presto, C. T., Sherman, M. F. \& Sherman, N. C. (1985). The effects of a masturbatory seminar on high school males' attitudes, false beliefs, guilt, and behavior. Journal of Sex Research, 21, 142-156.

Malo de Molina, C., Valls Blanco, J. M. \& Pérez Gómez, A. (1988). La conducta sexual de los españoles. Barcelona: Ediciones B.

Miller, W. R. \& Lief, H. (1976). Masturbatory attitudes, knowledge, and experience: Data from the Sex Kowledge and Attitude Test (SKAT). Archives of Sexual Behavior, 5, 447-467.

Montero, I. \& León, O. G. (2007). A guide for naming research studies in Psychology. International Journal of Clinical and Health Psychology, 7, 847-862

Mosher, D. L. (1998). Negative attitudes toward masturbation. En C. M. Davis, W. L. Yarber, R. Bauserman, G. Schreer \& S. L. Davis (Eds.), Handbook of sexuality-related measures (pp. 475-476). Londres: Sage.

Mosher, D. L. \& Abramson, P. R. (1977). Subjective sexual arousal to films of masturbation. Journal of Consulting and Clinical Psychology, 45, 796-807.

Mosher, D. L. \& Vonderheide, S. G. (1985). Contributions of sex guilt and masturbation guilt to women's contraceptive attitudes and use. Journal of Sex Research, 21, 24-39.

Nunnally, J. C. \& Bernstein, I. J. (1995). Teoría psicométrica. Madrid: McGraw-Hill.

Ortega, V., Ojeda, P., Sutil, F. \& Sierra, J.C. (2005). Culpabilidad sexual en adolescentes: estudio de algunos factores relacionados. Anales de Psicología, $21,268-275$.

Patton, M. S. (1986). Twentieth-century attitudes toward masturbation. Journal of Religion and Health, 25, 291-302.

Riley, A. J. \& Riley, E. J. (1978). A controlled study to evaluate masturbation in the management of 
primary orgasmic failure women. British Journal of Psychiatry, 133, 404-409.

Sierra, J. C. \& Buela-Casal, G. (2001). Evaluación y tratamiento de las disfunciones sexuales. En G. Buela-Casal \& J. C. Sierra (Eds.), Manual de evaluación y tratamientos psicológicos (pp. 439-485). Madrid: Biblioteca Nueva.

Sierra, J. C., Ortega, V. \& Gutiérrez-Quintanilla, J. R. (2008). Encuesta de Opinión Sexual: fiabilidad, validez y datos normativos de una versión reducida en muestras salvadoreñas. Revista Mexicana de Psicología, 25, 139-150.

Van der Hofstadt, C. J., Antón Ruiz, F. A., Tirado, S. \& Navarro-Cremades, F. (2007). Hábitos sexuales en mujeres estudiantes de medicina. Cuadernos de Medicina Psicosomátrica y Psiquiatría de Enlace, 84/85, 32-48.

Wang, R. J., Huang, Y. \& Lin, Y. C. (2007). A study of masturbatory kowledge and attitudes and related factors among Taiwan adolescents. Journal of Nursing Research, 15, 233-242.

World Association for Sexual Health. (1999). Declaración universal de los derechos sexuales. Recuperado el 6 de octubre, 2008, de http://www.worldsexology. org/esp/about_sexualrights_span.asp

\section{Anexo}

Versión española del Inventario de Actitudes Negativas hacia la Masturbación (Abramson \& Mosher, 1975)

Los siguientes 30 ítems muestran diversas opiniones y actitudes sobre la masturbación. Para contestar señale una de las 5 opciones de respuesta:

1 significa que el ítem es totalmente falso

2 significa bastante falso

3 significa indecisión

4 bastante verdadero

5 totalmente verdadero

1. La gente se masturba para escapar de sentimientos de tensión e inquietud.

2. La gente que se masturba no disfruta tanto el coito.
3. La masturbación es un asunto privado que ni ofende ni importa a los demás.

4. La masturbación es un pecado contra uno mismo.

5. La masturbación en la niñez puede ayudar a desarrollar una actitud saludable y natural hacia el sexo.

6. La masturbación en un adulto es juvenil e inmadura.

7. La masturbación puede conducir a la homosexualidad.

8. La masturbación excesiva es físicamente imposible, una idea absurda.

9. Si disfruta mucho masturbándose, no aprenderá nunca a relacionarse con el sexo opuesto.

10. Después de la masturbación, la persona se siente degradada.

11.La experiencia con la masturbación puede ayudar potencialmente a una mujer a tener orgasmos en el coito.

12. Me siento culpable masturbándome.

13.La masturbación puede ser "el amigo en la necesidad" cuando no hay "amigo en el acto".

14. La masturbación puede ser una salida para fantasías sexuales sin dañar a otros o ponerse en peligro uno mismo.

15.La masturbación en exceso puede conducir a problemas de impotencia en los hombres y frigidez en las mujeres.

16.La masturbación es un mecanismo de escape que impide desarrollar una perspectiva sexual madura.

17. La masturbación puede proporcionar un alivio inofensivo a la tensión sexual.

18.Jugar con los propios genitales es repugnante.

19. La masturbación excesiva se asocia con la neurosis, la depresión y problemas del comportamiento.

20.Cualquier masturbación es excesiva.

21.La masturbación es un acto compulsivo, hábito adictivo que una vez comenzado es casi imposible parar.

22. La masturbación es divertida.

23. Cuando me masturbo, me disgusto conmigo mismo. 
24. Un patrón de masturbación frecuente se asocia a la introversión y al aislamiento de los contactos sociales.

25. Me avergonzaría tener que admitir públicamente que me he masturbado.

26. La masturbación excesiva conduce al agotamiento y embotamiento mental.
27.La masturbación es un desahogo sexual normal. 28. La masturbación es ocasionada por una excesiva preocupación por el sexo.

29. La masturbación puede enseñar a disfrutar la sensibilidad del propio cuerpo.

30.Después de masturbarme me disgusto por la falta de control sobre mi cuerpo. 